\title{
Diferencias en la aterosclerosis coronaria entre hombres y mujeres con Diabetes Mellitus tipo 2 evaluados con el Score de Gensini
}

Luis Gajardo1, Eduardo Lecannelier1, Reinaldo Venegas1, Luis Pérez1, Álvaro Saldaña1, Gilda Vargas2A, Jessica Parra3B, Virginia Segall3B, Isabel Robles3B, Jorge Gajardo1C, Liliana Lamperti4DE.

\author{
1 Facultad de Medicina, C Estudiante Medicina, Universidad de Concepción \\ 2 Facultad de Ciencias, AMSc en Estadística, Universidad del Bío-Bío \\ 3 Centro Cardioquirúrgico, B Enfermera Universitaria, Hospital Regional de Concepción \\ 4 Facultad de Farmacia, D Bioquímico, E PhD, Universidad de Concepción \\ Trabajo financiado por Dirección de Investigación DIUC 209085032-1, Universidad de Concepción
}

Antecedentes y Objetivos: Las mujeres diabéticas tienen mayor morbi-mortalidad asociada a eventos coronarios isquémicos que los hombres. Este estudio describe la extensión, magnitud y severidad de la aterosclerosis coronaria entre hombres y mujeres con diabetes mellitus tipo 2.

Métodos: Estudio de tipo descriptivo que incluyó a 162 pacientes diabéticos consecutivos con sospecha de aterosclerosis coronaria y estudiados con coronariografía. La magnitud de la aterosclerosis fue cuantificada utilizando el Score de Gensini.

Resultados: La edad promedio fue 64.8 años. Noventa y cuatro eran mujeres (58.0\%). El tiempo de evolución de la diabetes fue mayor para mujeres que para hombres (152.1 \pm 90.0 vs $120.2 \pm 99.4$ meses $)$ respectivamente $(\mathrm{p}<0.05)$. Las mujeres tuvieron un índice de masa corporal $(30.5 \pm 5.1)$, superior al de los hombres $(28.5 \pm 5.2)(\mathrm{p}<0.05)$. La aterosclerosis coronaria significativa estuvo presente en el $61.7 \%$ de toda la población diabética, siendo en hombres de $76.5 \%$ versus $51.5 \%$ en mujeres (odds ratio: 1.5). El número de placas ateroscleróticas fue 195 en 68 hombres (2,86 placa/paciente) y de 168 en 94 mujeres ( 1,78 placa/paciente) $(\mathrm{p}<0.0043)$. La presencia de aterosclerosis significativa en 1,2 y 3 vasos se observó en el $7.4 \% ; 17.6 \%$ y $51.5 \%$ de los hombres, respectivamente, versus el $12.8 \% ; 10.6 \%$ y $27.7 \%$ en las mujeres, $(\mathrm{p}<0.0002)$. La magnitud de la enfermedad coronaria medida con el Score de Gensini fue $69.4 \pm 66.7$ en hombres versus $35.6 \pm 47.3$ en mujeres $(\mathrm{p}<0.005)$.

Conclusión: En este estudio observamos que hombres diabéticos tienen mayor extensión, magnitud y severidad de aterosclerosis coronaria que las mujeres diabéticas.

\section{Correspondencia:}

Dr. Luis Jorge Gajardo

Departamento de Medicina Interna

Facultad de Medicina

Universidad de Concepción.

Casilla 160 C, Concepción

Teléfono: (41)-2463962

Fax: (41) 2255700

Email: jgncardio@gmail.com, Igajardo@udec.cl 


\section{Differences in coronary atherosclerosis assessed with the Gensini score between men and women with type 2 Diabetes}

Diabetic women have higher morbidity and mortality associated with ischemic coronary events than diabetic men, but the extension of coronary artery disease in both groups is less well established.

Aim : to compare the extension, magnitude and severity of coronary atherosclerosis between men and women with type 2 diabetes mellitus.

Method: 162 consecutive diabetic patients with suspected coronary atherosclerosis studied by coronary angiography were included. The magnitude of atherosclerosis was quantified using the Gensini Score.

Results: The average age was 64.8 years. Ninetyfour patients were women $(58.0 \%)$. Diabetes was present for $152 \pm 90.0$ months for women and $120 \pm 99.4$ months for men $(\mathrm{p}<0.05)$. Women had a higher body mass index $(30.5 \pm 5.1)$ than men $(28.5 \pm 5.2, \mathrm{p}<0.05)$.
The presence of significant coronary atherosclerosis in the entire diabetic population was of $61.7 \%: 76.5 \%$ in men and $51.5 \%$ in women (OR 1.5). The mean number of atherosclerotic plaques was 195 in 68 men (2.86 plaques/patient) and 168 in 94 women (1.78 plaques/patient, $\mathrm{p}=0.0043$ ). Significant atherosclerosis in 1,2 and 3 vessels was observed in $7.4 \%, 17.6 \%$ and $51.5 \%$ of men, respectively, versus $12.8 \%, 10.6 \%$ and $27.7 \%$ in women ( $p<0.0002)$. The magnitude of coronary disease measured by Gensini Score was $69.4+66.7$ in men versus $35.6 \pm 47.3$ in women $(\mathrm{p}<0.005)$.

Conclusion: Men with diabetes have greater extension, magnitude and severity of coronary atherosclerosis than diabetic women.

Key words: Coronary angiography. Diabetes mellitus. Atherosclerosis

\section{Introducción}

La Diabetes Mellitus 2 (DM2) es una de las enfermedades con mayor impacto en salud pública, tanto por su elevada frecuencia, como por las complicaciones crónicas que produce y su importante papel como factor de riesgo de aterosclerosis ${ }^{1}$. De todos los pacientes diabéticos que mueren en los Estados Unidos, el $65 \%$ es por enfermedad cardiovascular $^{2}$, siendo la aterosclerosis la principal causa de muerte prematura en estos pacientes y tanto mujeres como hombres diabéticos son vulnerables a la enfermedad coronaria isquémica. Los diabéticos tienen 2-4 veces mayor riesgo de infarto del miocardio y de muerte cardiovascular que individuos sin diabetes ${ }^{3}$, de tal forma que este desorden metabólico constituye un factor de riesgo independiente de mayor morbilidad y mortalidad cardiovascular y probablemente uno de los más importantes para el desarrollo de la aterosclerosis coronaria ${ }^{4}$.

La morbi-mortalidad de la mujer diabética muestra un peor pronóstico en los cuadros coronarios agudos con tasas de mortalidad el doble que la del hombre diabético ${ }^{5} \mathrm{y}$ un riesgo de isquemia cardíaca 3.3 veces superior comparada con mujeres no diabéticas ${ }^{6}$. En general, el riesgo relativo para enfermedad coronaria fatal asociado con DM2 es mayor al $50 \%$ en mujeres que lo observado en hombres 7. Finalmente, para intentar explicar el mayor riesgo y peor pronóstico de las mujeres diabéticas, algunos autores han señalado que la mujer diabética tendría un perfil de riesgo cardiovascular más adverso, una peor respuesta metabólica y una posible disparidad en el tratamiento que lo observado en el hombre diabético ${ }^{8,9}$. Desde el punto de vista anatómico, en la población sin diabetes, se ha reportado que las mujeres tienen una menor frecuencia de coronariopatía obstructiva que el hombre, evaluada en la coronariografía ${ }^{10,11}$. Sin embargo, la extensión, magnitud y severidad de la aterosclerosis coronaria entre hombres y mujeres con DM2, estudiada por coronariografía invasiva no es conocida.

El objetivo fundamental de este estudio fue observar si existen diferencias en la presencia de aterosclerosis coronaria entre hombres y mujeres con DM2 y evaluar la extensión, magnitud y severidad y de ella a través del Score de Gensini

\section{Metodología}

\section{Tipo de estudio y selección de pacientes}

Estudio no experimental, descriptivo, efectuado en el Centro cardioquirúrgico del Hospital Clínico Regional de Concepción (HCRC) entre marzo de 2007 y marzo de 2009. El universo lo constituyeron pacientes con DM2, con al menos 5 años de evolución de su diabetes, en trata- 
miento con insulina y/o hipoglicemiantes orales, quienes acudieron al Centro cardioquirúrgico del HCRC, desde donde fueron derivados a coronariografía por sospecha clínica de enfermedad coronaria y/o pruebas no invasivas positivas para insuficiencia coronaria. Los criterios de exclusión fueron: Insuficiencia renal severa con tasa de filtración menor a $30 \mathrm{ml} / \mathrm{min}$; daño neurológico por accidente vascular cerebral; imposibilidad de efectuar estudio coronariográfico; alergia a medio de contraste; tratamiento con esteroides en forma crónica; presencia de enfermedad de tipo autoinmune; diabetes mellitus tipo 1 y pacientes sometidos a algún tipo de revascularización miocárdica previa.

La anamnesis, examen físico y un electrocardiograma basal fueron efectuados en la primera entrevista. Todos los pacientes fueron medidos y pesados. Se tomó una muestra sanguínea para análisis de perfil lipídico, glicemia y hemoglobina glicosilada A1c.

Todos los pacientes aprobaron su inclusión al estudio y firmaron el consentimiento informado.

\section{Protocolo de estudio coronariográfico}

El estudio coronariográfico se efectuó en un Angiógrafo marca Siemens. Los pacientes se hospitalizaron el mismo día del estudio y fueron dados de alta 24 horas más tarde. La vía de acceso fue femoral o radial de acuerdo al protocolo habitual que se utiliza en el Laboratorio de Hemodinamia. Se efectuó un mínimo de 9 proyecciones en cada estudio. Se consideró como estenosis significativa una reducción de la luz del vaso igual o superior al 50\% de su diámetro. El examen fue informado por el cardiólogo operador y la información quedó almacenada en disco compacto, además del informe escrito que fue archivado y respaldado en el mismo laboratorio.

\section{Cuantificación de la magnitud de la aterosclerosis coronaria. \\ Score de Gensini}

El Score de Gensini es un método de cuantificación global de la magnitud de la aterosclerosis coronaria previamente validado por Peppes y col $2008{ }^{12}$. Este Score se calcula a partir de la información entregada en la coronariografía de cada paciente. Se asignó un valor a cada estenosis coronaria según su severidad y su importancia topográfica, evaluándose en al menos dos proyecciones. Para estenosis del 25\%, 50\%, 75\%, 90\%, 99\%, y oclusiones completas les fueron asignados un valor de 1, 2, 4, 8, 16, y 32 respectivamente. A cada segmento vascular principal le fue asignado un factor multiplicador de acuerdo con la significancia funcional del área miocárdica suplida por ese segmento. De este modo al tronco común izquierdo le fue asignado el valor 5; a la descendente anterior izquierda proximal: 2.5 ; al segmento proximal de la arteria circunfleja: 2.5 ; al segmento medio de la descendente anterior: 1.5; a la arteria coronaria derecha, el segmento distal de la descendente anterior, la arteria posterolateral y la arteria obtusa marginal: 1 ; y otras: 0.5 . La evaluación del grado de estenosis de cada arteria fue efectuada en forma visual por al menos 2 observadores en forma independiente. $\mathrm{La}$ oclusión coronaria crónica fue definida como una obstrucción del $100 \%$ de la luz del vaso sin flujo anterogrado y con al menos 3 meses de antigüedad de la oclusión.

\section{Marcadores Bioquímicos}

Los niveles de glicemia y perfil lipídico fueron medidos según método fotométrico enzimático estandarizado (Roche) y la glicohemoglobina por el método estándar de HPLC (Cromatografía de alta eficiencia) (Menarini).

\section{Análisis estadístico:}

Para caracterizar la población objeto de estudio, se realizó un análisis de datos univariado para todas las variables clínicas, y para establecer comparaciones entre ambos géneros, para dichas variables se utilizaron pruebas de diferencia de proporciones y de diferencias de medias para las variables cualitativas y cuantitativas, respectivamente. Para cuantificar la presencia de enfermedad coronaria se determinó la proporción de individuos que presentaban aterosclerosis coronaria en relación al total, lo que permitió además estimar la probabilidad de riesgo de que un sujeto de esta población tenga la enfermedad. La probabilidad estimada de padecer aterosclerosis coronaria se determinó mediante el odds ratio.

Para la medición de la severidad de la aterosclerosis y comparación según género, se utilizaron técnicas de estadística no paramétrica, tales como pruebas chi-cuadrado de homogeneidad para dos poblaciones y para la evaluación del riesgo de no tener ateromatosis, se utilizó el odds ratio.

Para establecer la magnitud de la aterosclerosis entre hombres y mujeres, se comparó el score de Gensini, descrito en la metodología, aplicando la prueba t para poblaciones independientes.

En todas las pruebas, se consideró que la diferencia es estadísticamente significativa, si el valor p es inferior a 0.05 . Se utilizó el software estadístico SPSS versión 16.0.

\section{Resultados}

\section{Características de la población}

Un total de 162 pacientes consecutivos con DM2 fueron estudiados con coronariografía invasiva. Noventa y cuatro eran mujeres $(58.0 \%)$ y $68(42.0 \%)$ eran hombres. No 
hubo diferencias significativas en las edades promedios entre los hombres $(66 \pm 10)$ y las mujeres $(66 \pm 9)$. Del total de la población estudiada, el 76\% (123 pacientes) tenía cuadros coronarios estables y el $24 \%$ (39 pacientes) síndromes coronarios agudos, entre los cuales hubo 20 infartos agudos del miocardio.

Las mujeres presentaron un tiempo de evolución de DM2 mayor que los hombres, que en promedio fue de $152.1 \pm$ 90 versus $120.2 \pm 99.4$ meses respectivamente $(\mathrm{p}<0.05)$ y un índice de masa corporal $(30.5 \pm 5.1 \mathrm{Kg} / \mathrm{m} 2)$ significativamente mayor que el de los hombres $(28.5 \pm 5.2$ $\mathrm{Kg} / \mathrm{m} 2)(\mathrm{p}<0.05)$. Los niveles de hemoglobina glicosilada A1c promedio, estuvieron en la categoría de mal control metabólico para ambos géneros $(7.9 \pm 2.0 \%$ versus 8.0 $\pm 2.0 \%$ ), presentando el $30.6 \%$ de los hombres niveles de hemoglobina glicosilada en rangos adecuados para un buen control metabólico versus el $28 \%$ de las mujeres. La hipertensión arterial se presentó en el $85,3 \%$ de los hombres y en el $91,5 \%$ de las mujeres $(\mathrm{p}=0,2156)$ y la dislipidemia se observó en el 52,9\% de los hombres versus el $44,6 \%$ de las mujeres ( $\mathrm{p}=0,2991$ ). El $16.2 \%$ de los hombres tenía hábito tabáquico, versus tan sólo el $2.1 \%$ de las mujeres $(\mathrm{p}=0,0012)$.

\section{Descripción de la enfermedad coronaria}

La presencia de enfermedad coronaria significativa (estenosis $\geq 50 \%$ ) en la totalidad de la población estudiada fue de $61.7 \%$, siendo de $76,5 \%$ en hombres vs $51,5 \%$ en mujeres (Odds ratio: 1.5) (Tabla 1).

El número total de placas ateroscleróticas con estenosis igual o superior al 50\% observadas en la angiografía de 94 mujeres fue de 168 (1.78 placa/paciente) versus 195 placas ateroscleróticas en 68 hombres ( 2.86 placa/paciente) $(\mathrm{p}<0.0043)$. La arteria más frecuentemente comprometida tanto en hombres como en mujeres fue la descendente anterior (Tabla 1).

La presencia de enfermedad significativa en 1,2 y 3 vasos se observó en el $7.4 \%, 17.6 \%$ y $51.5 \%$ de los hombres respectivamente versus el $12.8 \%, 10.6 \%$ y $27.7 \%$ de las mujeres, siendo sólo el compromiso de tres vasos mayor para el género masculino $(\mathrm{p}<0.0002)$. Se encontraron 64 vasos con oclusión crónica total, lo que fue significativamente mayor en hombres $(57.3 \%)$ que en mujeres $(26.5 \%)(\mathrm{p}=0.0001)$, destacando un porcentaje importante de oclusión total en hombres menores de 50 años de edad.

\section{Score de Gensini}

El análisis de la magnitud de la enfermedad coronaria a través del Score de Gensini fue de $69.4 \pm 66.7$ para los hombres versus $35.6 \pm 47.3$ para las mujeres $(\mathrm{p}<0.005)$.
El Score de Gensini promedio en los síndromes coronarios agudos (SCA) fue significativamente superior a lo observado en cuadros coronarios estables $(\mathrm{p}<0.0001)$. Los hombres presentaron un Score de Gensini superior tanto en SCA como en cuadros coronarios estables $(\mathrm{p}<0.003)$ (Tabla 1).

\begin{tabular}{|c|c|c|c|}
\hline & $\begin{array}{c}\text { Hombres } \\
(n=68)\end{array}$ & $\begin{array}{c}\text { Mujeres } \\
(\mathrm{n}=94)\end{array}$ & Valor $p$ \\
\hline Oclusiones no significativas (< $\mathbf{5 0} \%)$, \% & 23,5 & 48,9 & 0.001 \\
\hline Oclusiones significativas ( $\geq \mathbf{5 0} \%), \%$ & 76,5 & 51,5 & 0,0010 \\
\hline$N^{0}$ placas totales (estenosis $\geq \mathbf{5 0} \%$ ) & 195 & 168 & \\
\hline Relación placas/paciente & 2,86 & 1,78 & 0.0043 \\
\hline$N^{0}$ pacientes con lesiones $\geq \mathbf{5 0} \%$ en; & $n(\%)$ & $n(\%)$ & \\
\hline 1 vaso & $5(7,4)$ & $12(12,8)$ & 0,2672 \\
\hline 2 vasos & $12(17,6)$ & $10(10,6)$ & 0,1988 \\
\hline 30 más vasos & $35(51,5)$ & $26(27,7)$ & 0,0002 \\
\hline$^{0}$ vasos con oclusión crónica & $39(57.3 \%)$ & $25(26.5 \%)$ & 0.0001 \\
\hline Oclusión crónica en menores de 50 años & $16(8.2 \%)$ & $1(5.9 \%)$ & 0.0006 \\
\hline SG Total promedio (DS) & $69,43(66,7)$ & $35,6(47,3)$ & 0,005 \\
\hline SG ACE promedio (DS) & $54,9(57,8)$ & $28,1(44,4)$ & 0,0018 \\
\hline SG SCA promedio(DS) & $107(73)$ & $69,6(46,5)$ & 0,003 \\
\hline \multicolumn{4}{|l|}{ SG (Score de Gensini) } \\
\hline \multicolumn{4}{|l|}{ DS (desviación estándar) } \\
\hline \multicolumn{4}{|l|}{ ACE (angina crónica estable) } \\
\hline SCA ( síndrome coronaria aguc & & & \\
\hline
\end{tabular}

\section{Discusión}

En nuestro estudio, la aterosclerosis coronaria en diabéticos estuvo presente en el $61.7 \%$ de los pacientes. Esto concuerda con lo publicado en la literatura, en que las cifras oscilan entre 60 y $66 \%{ }^{13,14}$. Aún cuando esta cifra parece elevada, se debe considerar que el diagnóstico de aterosclerosis coronaria en este estudio es del tipo angiográfico y no clínico. Debe considerarse además que nuestra investigación no fue realizada en la población general, sino que en una muestra de pacientes con DM2 que acudieron a atención clínica y que tenían algún grado de sospecha de enfermedad coronaria clínica o de laboratorio. La alta prevalencia de aterosclerosis coronaria observada en este estudio viene a corroborar la información publicada hasta ahora, sobre el impacto de la DM2 sobre la aterosclerosis coronaria ${ }^{13}$. Estudios que comparen la presencia de enfermedad coronaria en diabéticos según género son escasos hasta el momento. En nuestro estudio, los hombres diabéticos tuvieron mayor extensión de aterosclerosis coronaria que las mujeres, con una probabilidad estimada de padecer aterosclerosis coronaria de un $50 \%$ superior para los hombres.

En la población estudiada no hubo diferencias en la edad promedio entre ambos grupos y considerando que se ha 
LDL a $70 \mathrm{mg} / \mathrm{dl}$ (opcional).

\section{4.- Rol de los Triglicéridos (TG)}

Los triglicéridos (TG) han sido implicados en la génesis del AVE.

Un estudio prospectivo publicado recientemente de casi 14.000 pacientes seguidos por 33 años en Dinamarca, correlaciona niveles altos de TG sin ayuno (entre 289 y $442 \mathrm{mg} / \mathrm{dl}$ ) con alta incidencia de AVE, especialmente en el sexo femenino. No se encontró correlación con los niveles de colesterol, excepto con cifras de colesterol total sobre $348 \mathrm{mg} / \mathrm{dl}^{17}$.

Esto ha sido controversial, pues los TG están correlacionados fuertemente con diabetes, HDL bajo y la grasa corporal, factores de por si de riesgo cardiovascular, lo que en estudios poblacionales puede pesar mucho. En una revisión sistemática de Leonards ${ }^{18}$ se revisaron 25 estudios, 13 de cohorte y 12 de caso- control, algunos correlacionaron AVE con niveles de TG en ayunas y otros con TG sin ayuno, concluyendo que los datos son inconsistentes y se requiere estandarizar las técnicas de medición.

\section{Conclusiones}

La correlación directa entre niveles de colesterol total y AVE isquémico (trombótico) solo se ha podido demostrar con cifras cercanas a $300 \mathrm{mg} / \mathrm{dl}$ lo que no es habitual de ver. No hay estudios que muestren que subfracciones de colesterol puedan ser más influyentes.

Sin embargo, la terapia hipolipemiante con estatinas es la que ha mostrado mayores beneficios en la reducción de riesgo de AVE, y se le ha atribuido a sus efectos "pleiotrópicos" dado que la reducción del colesterol no lo ha mostrado. No se ha podido demostrar este beneficio con otros hipolipemiantes. ${ }^{15}$

Respecto a Triglicéridos, si bien hay estudios que los correlacionan con el AVE, el análisis de varios de ellos no ha podido ser consistente como para tomar conductas terapéuticas.

\section{Referencias:}

1.- MINISTERIO DE SALUD. Guía Clínica Ataque Cerebrovascular Isquémico del Adulto. Septiembre 2007.

2.- ARBOIX A, SÁNCHEZ E, BALCELLS M. Different vascular risk factor profiles in ischemic stroke versus intracerebral hemorrhage: a study in 1,702 consecutive patients with acute stroke. Med Clin (Barc). 2001 ;116:89-91.

3.- Cholesterol, diastolic blood pressure, and stroke: 13,000 strokes in 450,000 people in 45 prospective cohorts. Prospective studies collaboration. Lancet 1995;346:1647-53

4.- LEPPÄLÄ JM, VIRTAMO J, FOGELHOLM R, ALBANES D, HEINONEN OP. Different risk factors for different stroke subtypes: association of blood pressure, cholesterol, and antioxidants. Stroke. 1999;30:2535-40.

5.- $\quad$ SEVER PS, DAHLÖF B, POULTER NR, WEDEL H, BEEVERS G, CAULFIELD M, et al; ASCOT investigators. Prevention of coronary and stroke events with atorvastatin in hypertensive patients who have average or lower-than-average cholesterol concentrations, in the Anglo-Scandinavian Cardiac Outcomes Trial--Lipid Lowering Arm (ASCOT-LLA): a multicentre randomised controlled trial. Lancet. 2003; 361: 1149-1158.

6.- COLHOUN HM, BETTERIDGE DJ, DURRINGTON PN, HITMAN GA, NEIL HA, LIVINGSTON SJ, et al. Primary prevention of cardiovascular disease with atorvastatin in type 2 diabetes in the Collaborative Atorvastatin Diabetes Study (CARDS): multicentre randomised placebo-controlled trial. Lancet 2004;364:685-696

7.- LAROSA JC, GRUNDY SM, WATERS DD, SHEAR C, BARTER P, FRUCHART JC, et al. Intensive lipid lowering with atorvastatin in patients with stable coronary disease. N Engl J Med. 2005; 352:1425-35.

8.- AMARENCO P, LABREUCHE J, LAVALLÉE P, TOUBOUL PJ. Statins in stroke prevention and carotid atherosclerosis: systematic review and up-to-date meta-analysis. Stroke. 2004; 35:2902-9.

9.- The SPARCL Investigators. Stroke Prevention by Aggressive Reduction in Cholesterol Levels (SPARCL) Investigators. High-dose atorvastatin after stroke or transient ischemic attack. N Engl J Med. 2006; 355:549 -559.

10.- VIVANCOS-MORA J, GIL-NÚÑEZ AC. Lipids and stroke: the opportunity of lipid-lowering treatment. Cerebrovasc Dis 2005; 20: 53-67.

11.- ARBOIX A, GARCÍA-EROLES L, OLIVERES M, TARGA C, BALCELLS M, MASSONS J. Pretreatment with 
statins improves early outcome in patients with first-ever ischaemic stroke: a pleiotropic effect of statins or a beneficial effect of hypercholesterolemia? BMC Neurology 2010; 10:47

12.- ABOA-EBOUlÉ C, BINQUET C, JACQUIN A, HERVIEU M, BONITHON-KOPP C, DURIER J, et al. Effect of previous statin therapy on severity and outcome in ischemic stroke patients: a population-based study. J Neurol. 2012 . Published online 24 Jun 2012.

13.- EVERETT BM, GLYNN RJ, MACFADYEN JG, RIDKER

PM. Rosuvastatin in the prevention of stroke among men and women with elevated levels of C-reactive protein: justification for the Use of Statins in Prevention: an Intervention Trial Evaluating Rosuvastatin (JUPITER). Circulation 2010;121:143-50.

14.- SOBRINO T, BLANCO M, PÉREZ-MATO M, RODRÍGUEZ-YÁÑEZ M, CASTILLO J. Increased levels of circulating endothelial progenitor cells in patients with ischaemic stroke treated with statins during acute phase. Eur
J Neurol. 2012;10: 1468-1331.

15.- GOLDSTEIN LB, BUSHNELL CD, ADAMS RJ, APPEL LJ, BRAUN LT, CHATURVEDI S, et al. Guidelines for the primary prevention of stroke: a guideline for healthcare professionals from the American Heart Association/American Stroke Association. Stroke 2011; 42 :517-84.

16.- GRUNDY SM, CLEEMAN JI, MERZ CN, BREWER HB, JR, CLARK LT, HUNNINGHAKE DB, et al. Implications of recent clinical trials for the National Cholesterol Education Program Adult Treatment Panel III guidelines. J Am Coll Cardiol 2004;44:720-32.

17.- VARBO A, NORDESTGAARD BG, TYBJAERG-HANSEN A, SCHNOHR P, JENSEN GB, BENN M. Nonfasting triglycerides, cholesterol, and ischemic stroke in the general population.. Ann Neurol. 2011; 69:628-34.

18.- LEONARDS C, EBINGER M, BATLUK J, MALZAHN U, HEUSCHMANN P, ENDRES M. The role of fasting versus non-fasting triglycerides in ischemic stroke: a systematic review. Front Neurol. 2010; 1:133. 